\title{
Métodos de Tratamento Utilizados na Incontinência Urinária de Esforço Genuina: um Estudo Comparativo entre Cinesioterapia e Eletroestimulação Endovaginal
}

\begin{abstract}
Methods of Treatment of Genuine Stress Incontinence: a Comparative Study between a Pelvic
\end{abstract} Floor Exercise Program and a Pelvic Floor Electrical Stimulation

Nicole O. Bernardes, Flávia R. Péres, Elza L.B.L. Souza, Oswaldo L.Souza

\begin{abstract}
RESUM0
Objetivos: comparar pacientes portadoras de incontinência urinária de esforço genuina (IUEG) que se submeteram a um protocolo de cinesioterapia para reforço perineal àquelas que se submeteram a eletroestimulação endovaginal (EEEV). O objetivo foi traçar uma conduta de tratamento fisioterápico mais adequada para tal enfermidade.

Métodos: foram selecionadas 14 pacientes portadoras de IUEG, com idade entre 31 e 64 anos, sendo divididas em dois grupos de 7 mulheres cada. Cada grupo foi submetido a um dos protocolos de tratamento ambulatorial diário durante 10 dias consecutivos. As pacientes foram avaliadas e reavaliadas por somente uma fisioterapeuta. Para as análises estatísticas foram utilizados testes não-paramétricos.

Resultados: todas pacientes obtiveram uma melhora parcial ou total da IUEG com 10 sessões fisioterápicas consecutivas, considerando os critérios objetivos e subjetivos de avaliação.

Conclusão: tanto a cinesioterapia quanto a EEEV se mostraram efetivas no tratamento da IUEG, porém, a cinesioterapia para reforço perineal apresentou uma tendência para ser o tratamento de escolha.
\end{abstract}

PALAVRAS-CHAVE: Incontinência urinária de esforço genuína. Cinesioterapia. Eletroestimulação endovaginal.

\section{Introdução}

Segundo a "International Continence Society", incontinência urinária de esforço genuína (IUEG) é a perda involuntária de urina pela uretra, ocorrida quando a pressão intravesical exceder a pressão uretral máxima na ausência de contração do músculo detrusor ${ }^{1}$. A forma de incontinência urinária que apresenta maior prevalência é a IUEG, sendo responsável por $60 \%$ de todos os casos de incontinência urinária femi-

Santa Casa de Belo Horizonte - Belo Horizonte - MG

Correspondencia:

Nicole de Oliveira Bernardes

Rua Alegrete 253/102 - Sagrada Família

31035-380 - Belo Horizonte - MG nina. As perdas urinárias ocorrem quando há um aumento da pressão intra-abdominal por esforços tais como: tosse, espirro, risada, saltar, caminhar ou orgasmo ${ }^{2}$.

O tratamento é constituido por 2 modalidades: conservador e cirúrgico. O tratamento cirúrgico não é efetivo em todos os casos, além do que ocorre recidiva dos sintomas antes de 5 anos.

Um assoalho pélvico com função deficiente ou inadequada é um fator etiológico relevante na ocorrência da IUEG. Estudos recentes evidenciam que procedimentos fisioterápicos resultam no fortalecimento dessa musculatura, promovendo a continência urinária ${ }^{1}$.

Segundo Fortini e Rocha ${ }^{1}$, o exercício do assoalho pélvico é uma forma de tratamento para pacientes portadoras de IUEG. Tal abordagem ade- 
quadamente conduzida e supervisionada alcança resultados satisfatórios.

Hahn et al. ${ }^{3}$, Bo et al. ${ }^{4}$ e Holley et al. ${ }^{2}$ estão de acordo que a cinesioterapia é um tratamento efetivo. Em seus trabalhos citam a motivação da paciente como sendo uma importante parte para o sucesso do tratamento. A cinesioterapia tem determinado a melhora ou a cura de várias pacientes com efeito duradouro por mais de 5 anos.

Assim também, a eletroestimulação endovaginal (EEEV) tem mostrado resultados promissores. Pode-se dizer que é capaz de reeducar o assoalho pélvico; porém para melhores resultados, deve ser associada a outros métodos de tratamento conservador ${ }^{5,6}$.

O assoalho pélvico é composto por fibras musculares de contração lenta ou tipo I e rápida ou tipo II, sendo que $70 \%$ dessas são de contração lenta e responsáveis pela manutenção do tônus; os outros $30 \%$ são fibras de contração rápida e de baixa resistência ${ }^{7}$. Na IUEG as fibras mais acometidas são as de contração rápida.

A contração voluntária do assoalho pélvico age mais especificamente nas fibras do tipo II, fazendo com que elas se hipertrofiem, potencializando a força de contração perineal.

Vários trabalhos mostram a efetividade da cinesioterapia e da EEEV para o reforço muscular do assoalho pélvico. Ambas tratam a IUEG provocando contrações voluntárias dessa musculatura, estimulando as fibras do tipo I e II. Sabendo que a fraqueza e abaulamento do assoalho pélvico são fatores desencadeantes da sintomatologia da IUEG, a fisioterapia torna-se mais um recurso terapêutico que utiliza modalidades fisicas eficientes no tratamento dessa patologia ${ }^{8}$. Essa é a base da presente pesquisa.

O objetivo dessa investigação foi traçar uma conduta de tratamento fisioterápico mais adequada para pacientes portadoras de IUEG, sendo elas submetidas ou a um protocolo de cinesioterapia para reforço perineal ou a um de EEEV.

\section{Pacientes e Métodos}

No período de fevereiro a agosto de 1998, foram avaliadas no setor de fisioterapia aplicada à uroginecologia e obstetrícia da Santa Casa de Belo Horizonte 21 mulheres com idade entre 31 e 67 anos e diagnóstico médico de incontinência urinária. Deste total, foram recrutadas as pacientes portadoras de IUEG diagnosticada por meio de urodinâmica e/ou cistometria. Foram excluídas as mulheres que estavam grávidas, em uso de dispositivo intra-uterino (D.I.U.), em uso de marcapasso, que apresentavam doenças urológicas associadas ou doenças ortopédicas do ombro visto que determinados exercícios do protocolo cinesioterápico envolviam movimentos desta articulação, que apresentavam classificação de IUEG superior ao grau 3 de força muscular do assoalho pélvico, que não cumpriram os protocolos propostos e aquelas que realizaram tratamento fisioterápico para IUEG anterior ao início da pesquisa. Os critérios de inclusão foram os seguintes: pacientes portadoras de IUEG com grau de força de contração perineal igual a 1 , 2 ou 3, independente de fatores tais como: tratamento cirúrgico prévio para IUEG ou uso de hormonioterapia. Da amostra total, foram então selecionadas 14 mulheres.

Inicialmente, as pacientes foram esclarecidas sobre o tratamento e a pesquisa, sendo que todas concordaram em participar e assinaram um termo de permissão e compromisso.

$\mathrm{Na}$ avaliação fisioterápica, foi colhida a história uroginecológica pregressa e feito o exame físico, que constava de exame postural ${ }^{9}$ e classificação do grau de força muscular do assoalho pélvico. Esta classificação se processou da seguinte maneira: visualização da genitália externa, verificando-se a presença ou ausência de contração voluntária visível da musculatura do assoalho pélvico após comando verbal; palpação feita por meio do toque bidigital seguida de uma solicitação verbal à paciente para que contraísse a vagina apertando o dedo do examinador; após a visualização e a palpação classificou-se funcionalmente o assoalho pélvico segundo $\operatorname{Ortiz}^{10}(\mathrm{Ta}-$ bela 1); fez-se então o teste do dispositivo intravaginal (D.I.V.), utilizando-se Femtones [cones vaginais fabricado pela Convatec (U.S.A)]. Trata-se de um estojo com cinco cones de tamanho e forma idênticos, mas de pesos crescentes e numerados $\left(n^{\circ} .1=20 \mathrm{~g} ; n^{\circ} .2=32,5 \mathrm{~g} ; \mathrm{n}^{\circ} .3=45\right.$ g; $\left.\mathrm{n}^{\circ} .4=57,2 \mathrm{~g} ; \mathrm{n}^{\circ} .5=70 \mathrm{~g}\right)$. O primeiro cone utilizado no teste, selecionado por meio da avaliação funcional do assoalho pélvico (Tabela de Orti $z^{10}$ ), foi introduzido na vagina da paciente estando ela em decúbito dorsal com os quadris e joelhos fletidos, deixando-se o fio de náilon para fora para possibilitar sua remoção após o exame. A paciente foi submetida a uma seqüência crescente de esforços que são padronizados no serviço (caminhar durante 1 minuto; subir/descer escadas durante 30 segundos; pular, agachar/ levantar, tossir 4 vezes). Caso o cone continuasse no canal vaginal, esse era removido e se inseria o próximo de maior peso e assim por diante, até que algum deles se exteriorizasse aos esforços. Classificou-se então o grau de força do assoalho pélvico de acordo com o peso do cone sustentado. 
Tabela 1 - Classificação funcional do assoalho pélvico de acordo com a presença de contração voluntária (Ortiz, 1994) ${ }^{10}$

\begin{tabular}{ccc}
\hline Grau & Visualização & Palpação \\
\hline 0 & ausente & ausente \\
1 & ausente & reconhecível \\
2 & débil & reconhecível \\
3 & presente & sem resistência \\
4 & presente & com resistência não-mantida \\
5 & presente & com resistência mantida
\end{tabular}

O critério de avaliação obedeceu a uma seqüência rigorosa para todas as pacientes e o processo de avaliação física ficou a cargo de apenas uma fisioterapeuta, garantindo maior fidedignidade aos resultados.

Após a classificação, aplicaram-se aleatoriamente os protocolos de tratamento, sendo que sete pacientes submeteram-se ao protocolo de EEEV e as sete demais, ao de cinesioterapia para reforço perineal.

A EEEV foi realizada utilizando-se o aparelho Dualpex 961 da Quark Produtos Médicos com o eletrodo perineal intracavitário com dois anéis de metal que era introduzido com o auxílio do Gel Lubrificante KY da Johnson-Johnson. O programa de reforço de períneo escolhido foi o P54, por apresentar uma corrente bidirecional simétrica ${ }^{11}$, com os seguintes parâmetros: freqüência $(\mathrm{f})=30 \mathrm{~Hz}$, largura de pulso $(\mathrm{t})=1 \mathrm{~ms}$, tempo de sustentação (sust.) $=6 \mathrm{~s}$, tempo de repouso (rest.) $=2 \mathrm{x}$ tempo de sust., duração da terapia (time) $=20 \mathrm{~min}$.

Primeiramente, fez-se uma sessão experimental no dia da avaliação inicial, com o objetivo de testar a tolerância da paciente ao tratamento. Posteriormente, foram feitas dez sessões nos dias subseqüentes utilizando-se o mesmo programa (P54), porém alterando-se a freqüência para $60 \mathrm{~Hz}$, com a finalidade de provocar uma contração mais potente do assoalho pélvico. A intensidade utilizada foi de acordo com o limiar de cada paciente, observando-se a contração muscular, variando de 10 a $30 \mathrm{~mA}$. Após este período, foi feita uma reavaliação nos mesmos moldes da avaliação inicial, num prazo máximo de $24 \mathrm{~h}$ após a última sessão.

Do mesmo modo, o protocolo de cinesioterapia iniciou-se com uma sessão experimental no dia da avaliação inicial, constando de uma série de seis exercícios ${ }^{12}$ ( 2 específicos para musculatura abdominal, 2 específicos para assoalho pélvico e 2 associando contrações do assoalho pélvico, musculatura adutora e glútea), testando-se a adaptação da paciente ao programa. Nas dez sessões seguintes foram feitas três repetições da mesma série de exercícios, sustentando a contração voluntária do assoalho pélvico por seis segundos e repetindo-se
10 vezes cada exercício. Além disso, foi passado um programa domiciliar (controle da micção, reforço perineal) para ser feito diariamente durante o tratamento. Terminado o protocolo, as pacientes foram reavaliadas conforme a avaliação inicial, não ultrapassando $24 \mathrm{~h}$ após a última sessão.

\section{Resultados}

Este estudo teve como objetivo avaliar o comportamento de pacientes com IUEG usando dois tratamentos distintos: cinesioterapia e EEEV. Para tanto, 7 pacientes foram submetidas a cinesioterapia e outras 7 a EEEV. As comparações entre os tratamentos no que diz respeito a idade e ao peso foram realizadas utilizando-se o teste de KruskalWallis ${ }^{13,14}$. Este teste tem como objetivo comparar amostras independentes em relação a uma medida e trata-se de um teste não-paramétrico. Ressalta-se que se optou por testes não-paramétricos devido ao número reduzido de indivíduos. Todos os resultados foram considerados significativos para uma probabilidade de significância inferior a $5 \%$ $(\mathrm{p}<0,05)$, tendo-se portanto, pelo menos $95 \%$ de confiança nas conclusões apresentadas.

$A$ idade das pacientes que foram submetidas a EEEV variou de 45 a 64 anos (média $=53,3$ ) e no grupo de cinesioterapia houve uma variação de 31 a 59 anos (média=44,1). Entretanto esta diferença não se mostrou significativa. Também não foram constatadas diferenças significativas entre os dois grupos de pacientes no que se refere ao peso $(p=0,064)$. Para a EEEV verificou-se uma variação de 49 a 90 quilos e para a cinesioterapia a variação foi de 55,3 a 78 quilos. No que diz respeito à paridade, verificou-se apenas uma paciente nulipara no grupo que foi submetido a EEEV. As demais pacientes desse grupo e do grupo submetido a cinesioterapia eram multiparas.

A força de contração perineal foi medida antes do tratamento proposto e verificou-se no grupo submetido a EEEV que $85,7 \%$ dos casos tinham força " 0 " e $14,3 \%$ força igual a 1 . No grupo submetido a cinesioterapia, $57,1 \%$ dos casos tinham força " 0 ", $28,6 \%$ tinham força " 1 " e 14,2\% força "3". Já nas avaliações feitas após os protocolos de tratamento, verificou-se no grupo que foi submetido a EEEV que as pacientes apresentaram no máximo uma força igual a 2. No grupo submetido a cinesioterapia verificou-se que a maioria das pacientes apresentaram uma força superior a $2(57,2 \%)$ (Figura 1$)$. Considerando-se a classificação da perda urinária das pacientes portadoras de IUEG antes do tratamento, verificou-se no grupo de pacientes que foram submetidas a EEEV, 28,6\% dos casos com perda leve (perdas durante tosse forte, espirro, levantamento de peso ou exercícios bruscos) e 71,4\% com perda 
moderada (perdas durante qualquer tipo de tosse, riso ou atividades da vida diária). No grupo que foi submetido a cinesioterapia houve $42,9 \%$ dos casos com perda leve e $57,1 \%$ dos casos com perda moderada. Após o tratamento, verificou-se que no grupo de pacientes que foram submetidas a cinesiotera- pia, houve $71,4 \%$ de pacientes sem sintomas e $28,6 \%$ com perda leve. No grupo que foi submetido a EEEV houve $28,6 \%$ de pacientes sem sintomas, $57,1 \%$ com perda leve e $14,3 \%$ com perda moderada (Figura 2).

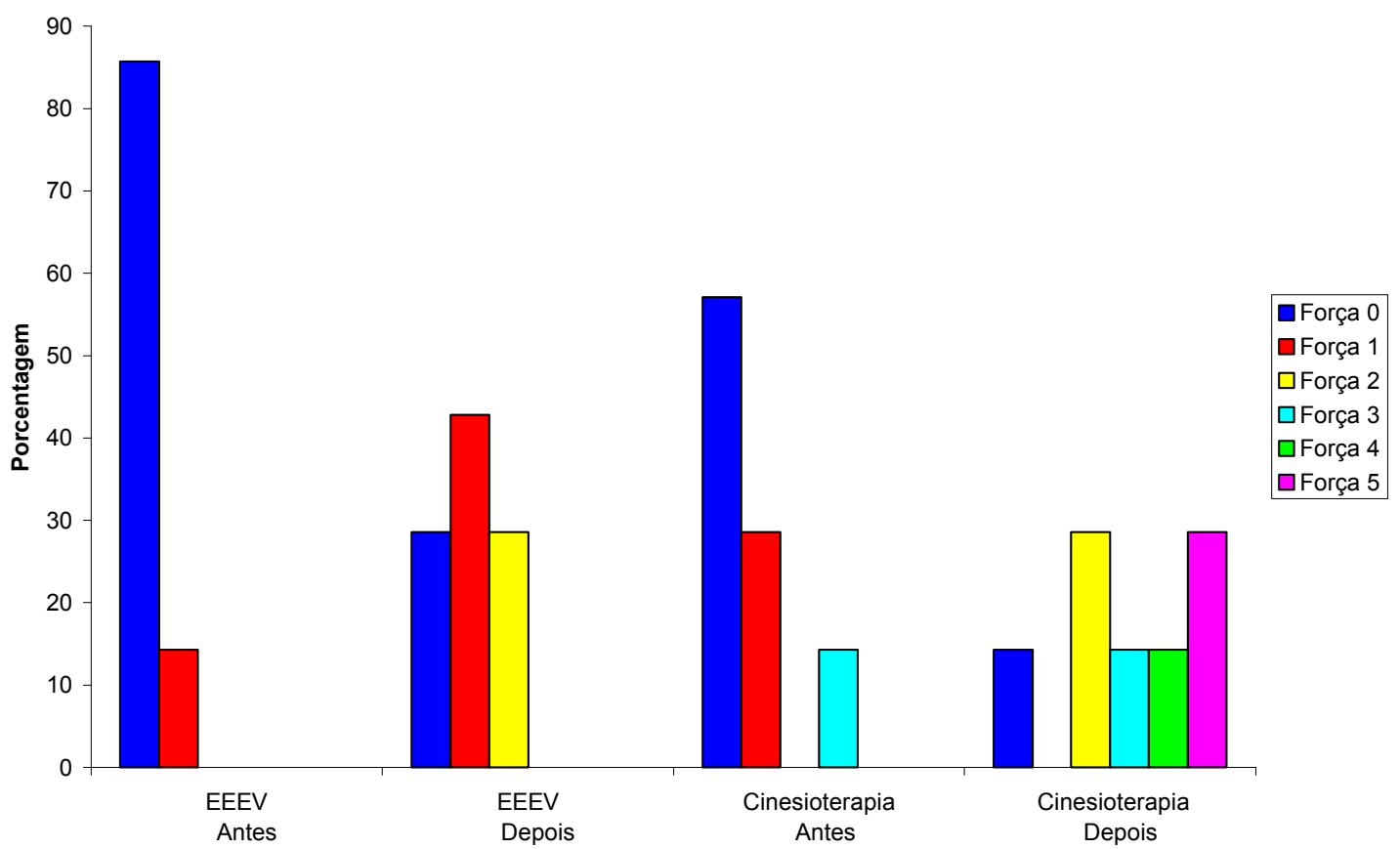

Figura 1 - Caracterização das pacientes quanto à força de contração perineal antes e depois do tratamento, considerando-se o tratamento proposto.

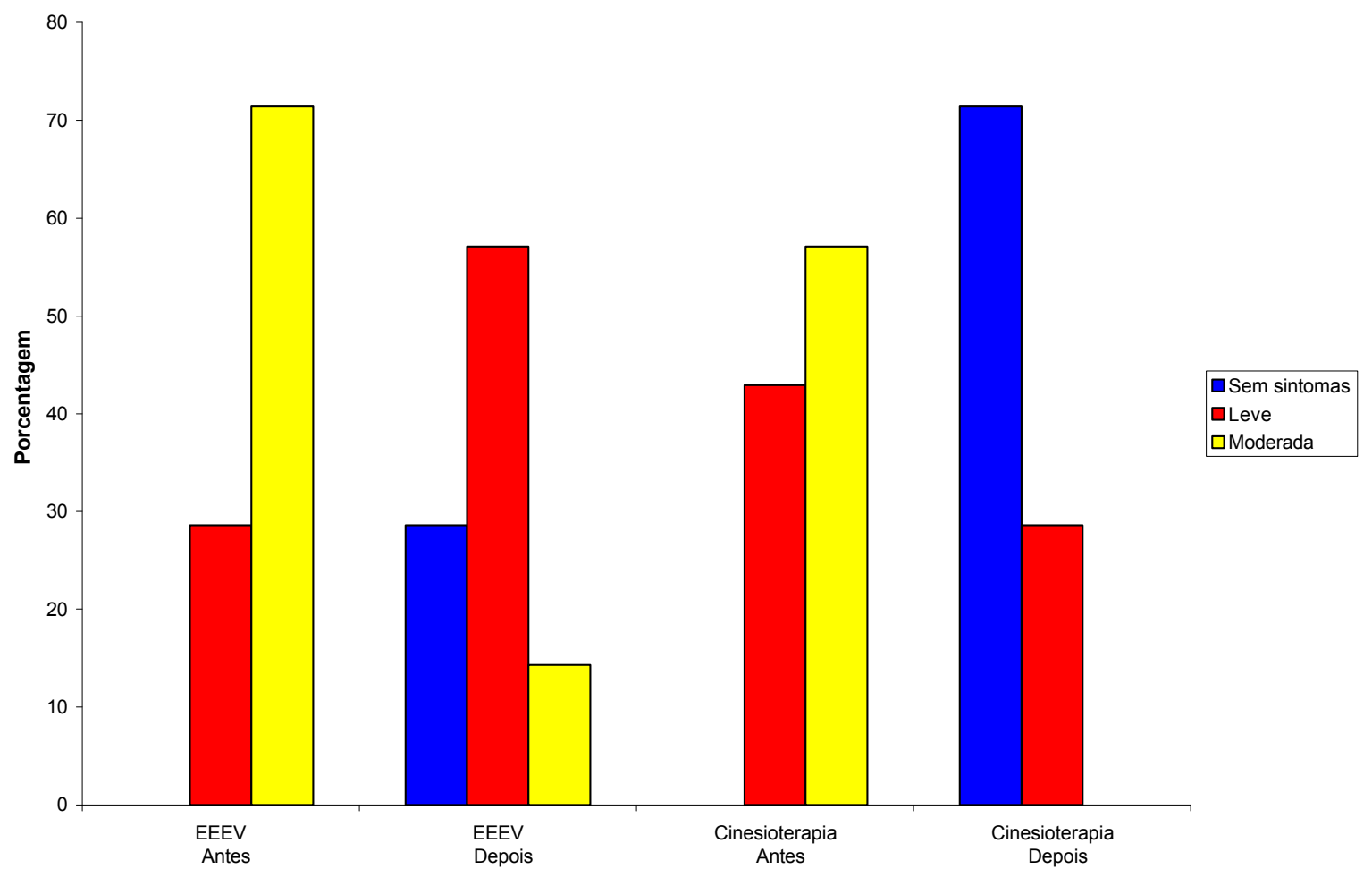

Figura 2 - Caracterização das pacientes com IUEG quanto à classificação da perda urinária antes e após o tratamento, considerando-se o tratamento proposto. 


\section{Discussão}

De acordo com os resultados obtidos pode-se concluir que a cinesioterapia apresenta uma tendência a ser o tratamento de escolha para IUEG leve ou moderada. Apesar de as pacientes tratadas pela EEEV terem obtido uma melhora considerável, a cinesioterapia apresentou melhores resultados em termos numéricos, considerando os protocolos de atendimento diário. Das 14 pacientes estudadas, 9 apresentavam IUEG moderada e 5 IUEG leve. Após o término do tratamento, 7 evoluíram sem sintomas, 6 com IUEG leve e apenas 1 com IUEG moderada. Esta análise subjetiva foi associada ao método objetivo de avaliação da força de contração perineal de acordo com o uso do teste do D.I.V., que vem confirmar este fato.

Esses resultados estão de acordo com relatos de outras pesquisas. Segundo Bo e Talseth ${ }^{15}$; Cammu e $\operatorname{Van}^{16}$ e Hahn et al. ${ }^{17}$, a cinesioterapia para reforço perineal produz uma melhora substancial ou a cura da IUEG; devendo-se associar critérios de avaliação subjetivos e objetivos, já que são usadas diferentes variáveis, durações, intensidades e freqüências nos treinamentos, tornando a comparação entre os estudos difícil. Para Smith ${ }^{18}$, Richardson et al. ${ }^{19}$ e Sand et al. ${ }^{20}$ a EEEV também é um tipo de tratamento conservador efetivo. Da mesma forma eles associam critérios subjetivos e objetivos para a avaliação, já que os parâmetros da EEEV são da mesma forma muito variáveis. O resultado deste estudo pode ser explicado pela fisiologia muscular do assoalho pélvico ${ }^{21}$. Estes músculos são histologicamente idênticos aos estriados esqueléticos e dessa forma possuem fibras lentas do tipo I e rápidas do tipo II A e $\mathrm{B}^{22}$. Trabalhando-se as fibras do tipo II com uma sobrecarga, acontece um aumento no volume dessas fibras, ou seja, ocorre uma hipertrofia muscular, que irá fazer com que a pressão de fechamento uretral permaneça positiva (pressão intrauretral > pressão intravesical), promovendo a continência urinária. A uretra possui um importante papel nesse mecanismo. Ela é composta por 3 camadas de musculatura: a interna (lisa) e duas camadas de externa (estriada). A musculatura estriada sobrepõe-se à lisa, havendo uma somatória de suas atividades. O esfincter externo ou estriado possui uma porção para-uretral que é composta de fibras de contração lenta e está em contato com a uretra e que em atividade permanente mantém o tônus. A outra porção pertence ao músculo elevador do ânus e produz contrações voluntárias das fibras tipo II.

Análises adicionais dos resultados sugerem que a diferença de idade entre os grupos não se apresentou significativa do ponto de vista estatístico; eles eram homogêneos. Porém, clinicamente falando, essa diferença pode ser representativa, considerando que o grupo selecionado aleatoriamente para a cinesioterapia apresentava idades menores que o grupo selecionado aleatoriamente para a EEEV. Vários fatores devem ser considerados quando se analisa a idade dessas pacientes: hormonioterapia, climatério, doenças ortopédicas, cardiopatias, disposição para a execução dos protocolos associada à motivação destas pacientes e quantidade de urina perdida pelas mesmas (o grupo de EEEV apresentou niveis de perda menores que o de cinesioterapia).

Esse experimento apresentou algumas falhas durante a sua execução. O número de pacientes foi reduzido, o que dificultou uma conclusão estatística mais definida se considerarmos a grande quantidade de variáveis selecionadas para essa pesquisa. Das 21 pacientes selecionadas 7 não realizaram os protocolos corretamente, devido ao alto custo dos exames complementares (urodinâmica e/ou cistometria), já que a grande maioria das pacientes era de baixo nivel socioeconômico. Esta baixa renda dificultou a realização diária dos protocolos. Um último fator a ser considerado é a disponibilidade dessas pacientes para o cumprimento dos protocolos ambulatoriais e domiciliares durante 10 sessões consecutivas. Dessa forma pesquisas que preencham essas lacunas se fazem necessárias para que se prove com embasamento estatístico e clínico a relevância da cinesioterapia e da EEEV como tratamentos conservadores eficientes e de menor custo que os tratamentos cirúrgicos.

Pesquisas revelam que pacientes que se tornaram continentes após a realização de protocolos de cinesioterapia e/ou EEEV voltaram ao quadro inicial de IUEG após 5 anos sem a manutenção destas terapias; portanto, os profissionais que prescrevem a reeducação do assoalho pélvico, como um tratamento conservador eficiente, devem enfatizar para a paciente que a manutenção da continência urinária depende da motivação e realização dos exercícios e/ou da EEEV por um minimo de três sessões ambulatoriais por semana, além de sessões domiciliares diárias.

\section{SUMMARY}

Purpose: to compare patients with genuine stress incontinence (GSI) who were submitted to a pelvic floor exercise program (PFEP) with those who were submitted to 
a pelvic floor electrical stimulation (PFES).

Methods: fourteen GSI patients, with age between 31-64 years, participated in the study. They were divided into two groups of 7 each. PFEP or PFES was performed for 10 days. The women were evaluated at the first consultation and reevaluated after the ten-day treatment by only one physical therapist. For the statistical analysis nonparametric tests were used.

Results: all had a partial or a total improvement of the GSI after the treatment, considering the subjective and objective analysis of the research.

Conclusion: both PFEP and PFES showed to be effective to treat GSI, although PFEP showed a tendency to be the better treatment.

KEY WORDS: Genuine stress incontinence. Pelvic floor exercise program. Pelvic floor electrical stimulation program.

\section{Agradecimentos:}

À fisioterapeuta Sílvia Elizate Monteiro, às médicas Márcia Salvador Géo, Rachel Silviano Brandão C. Lima e Tarina Marques Rubinger e aos médicos residentes em Ginecologia e Obstetricia da Santa Casa de Belo Horizonte.

\section{Referências}

1. Fortini A, Rocha R. Tratamento cinesioterápico da incontinência urinária genuína por stress. [Monografia]. Belo Horizonte: Escola de Educação Física da UFMG; 1996. p. 13-30, 37-53.

2. Holley RL, Varner RE, Kerns DJ, Mestecky PJ. Longterm failure of pelvic floor musculature exercises in treatment of genuine stress incontinence. South Med J 1995; 88:547-9.

3. Hahn I, Milsom I, Ohlsson Bl, Uhlemann C, Fall M, Comparative assessment of pelvic floor function using vaginal cones, vaginal digital palpation and vaginal pressure measurements. Gynecol Obstet Invest 1996; 41: 269-74.

4. Bo K, Maanum M. Does vaginal electrical stimulation cause pelvic floor muscle contraction? A pilot study. Scand J Urol Nephrol Suppl 1996; 179: 39-45.

5. Amaro JL, Agostinho AD, Trindade JCS, Lautenschiager MFM, Gasmeiro MOO. Eletroestimulação endovaginal no tratamento da incontinência urinária de esforço. J Bras Ginecol 1997; 107: 189-95.
6. Schussler B. Pelvic floor re-education: principles and practice. London: Springer-Verlag; 1994. p. 7-34, 37-98, 119-76.

7. Hollinshead HW. Anatomia. $4^{\mathrm{a}}$ ed. Rio de Janeiro: Interlivros; 1991. p. 625-71.

8. Polden M. Fisioterapia em ginecologia e obstetrícia. $1^{\text {a }}$ ed. São Paulo: Editora Santos; 1993. p. 33586.

9. Gould III JA. Fisioterapia na ortopedia e na medicina do esporte. $2^{\text {a }}$ ed. São Paulo: Manole; 1993. p. 67-68, 82, 217, 222-3.

10.Ortiz OC, Nudez FC, Ibañez G. Evaluación funcional del piso pelviano feminino (Classificación funcional). Bol Soc Lat Urog Cir Vag 1996; 1: 5-9.

11.Crépon F. Eletrofisioterapia e reeducação funcional. São Paulo: Lovise; 1996. p. 87, 89.

12.Lehmkuhl L. Cinesiologia clínica. $4^{\mathrm{a}}$ ed. São Paulo: Manole; 1989. p. 113-6, 267-92.

13. Conover WJ. Practical nonparametric statistics. New York: John Wiley \& Sons; 1980. p.224-6.

14.Johnson R, Bhattacharyya G. Statistics principles and methods. New York: John Wiley \& Sons; 1986.

15.Bo K, Talseth T. Long-term effect of pelvic floor muscle exercise 5 years after cessation of organized training. Obstet Gynecol 1996, 87: 2615.

16.Cammu H, Van NM. Pelvic floor muscle exercises: 5 years later. Urology 1995; 45:113-8.

17.Hahn I, Milsom I, Fall M, Ekelund P. Long-term results of pelvic floor training in female stress urinary incontinence. Br J Urol 1993; 72: 421-7.

18.Smith JJ $3^{\text {rd }}$. Intravaginal stimulation randomized trial. J Urol 1996; 155: 127-30.

19.Richardson DA, Miller KL, Sigel SW, Karram MM, Blackwood NB, Staskin DR. Pelvic floor electrical stimulation: a comparison of daily and everyother-day therapy for genuine stress incontinence. Urology 1996;48:110-8.

20.Sand PK, Richardson DA, Staskin DR, Swift SE, Appell RA, Whitmore KE et al. Pelvic floor electrical stimulation in the treatment of genuine stress incontinence: a multicenter, placebo-controlled trial. Am J Obstet Gynecol 1995; 173: 72-9.

21.Guyton A C. Tratado de fisiologia médica. 9a ed. Rio de Janeiro: Guanabara-Koogan; 1997. p.371-3.

22.Junqueira LC. Histologia básica. $8^{\mathrm{a}}$ ed. Rio de Janeiro: Guanabara-Koogan; 1997. p. 159-77. 